\title{
Epistatic Interactions between CREB and CREM Variants in Affective Disorder
}

\author{
Alberto Chiesa ${ }^{1,2}$, Agnese Marsano', Changsu Han ${ }^{3}$, Soo-Jung Lee ${ }^{4}$, \\ Ashwin A. Patkar ${ }^{5}$, Chi-Un Pae ${ }^{4,5}$, and Alessandro Serretti ${ }^{1}$ \\ ${ }^{1}$ Department of Biomedical and NeuroMotor Sciences, University of Bologna, Bologna, Italy \\ ${ }^{2}$ Department of Clinical and Experimental Medicine, University of Messina, Messina, Italy \\ ${ }^{3}$ Department of Psychiatry, College of Medicine, Korea University, Seoul, Republic of Korea \\ ${ }^{4}$ Department of Psychiatry, Bucheon St. Mary's Hospital, The Catholic University of Korea College of Medicine, Bucheon, Republic of Korea \\ ${ }^{5}$ Department of Psychiatry and Behavioral Sciences, Duke University Medical Center, Durham, NC, USA
}

\begin{abstract}
The aim of the present work is to investigate the existence of epistatic interactions possibly influencing psychotropic agents' response between rs6740584 within Cyclic adenosine monophosphate Response Element Binding (CREB) and rs12775799 within cAMP response element-modulator (CREM) variants in bipolar disorder (BD) and major depressive disorder (MDD). All BD and MDD patients were administered with the Young Mania Rating Scale (YMRS) and Hamilton Depression Rating Scale (HAMD) at baseline and at endpoint, respectively. A multiple regression model was employed to investigate the existence of possible epistatic interactions between the two variants and diverse clinical factors including drug response in affective disorders. No significant epistatic interaction was observed between rs6740584 within CREB and rs12775799 within CREM on both symptom improvement and other clinical factors in affective disorders. Our preliminary results suggest that no epistatic interaction between rs6740584 within CREB and rs12775799 within CREM should exist on clinical improvement and clinical factors in affective disorders.

Psychiatry Investig 2014;11(2):200-203
\end{abstract}

Key Words CREB, CREM, Epistasis, Response affective disorder.

\section{INTRODUCTION}

An increasing number of studies have recently focused on the role of the transcription factor cyclic adenosine monophosphate Response Element Binding (CREB) protein in mood disorders such as bipolar disorder (BD). Indeed, CREB plays a key role into neuronal signal transduction and it has been implicated in synaptogenesis, neural connections, and long term potentiation. ${ }^{1}$ Interestingly, genetic variations within the gene coding for CREB (CREB1) have been associated with treatment outcomes both in patients suffering from major depressive disorder $(\mathrm{MDD})^{2}$ and in patients suffering from $\mathrm{BD} .^{3} \mathrm{In}$ addition, CREB is thought to interact with cAMP response element-modulator (CREM), a protein that is highly homolo-

Received: April 4, 2013 Revised: May 20, 2013

Accepted: May 20, 2013 Available online: April 11, 2014

$\triangle$ Correspondence: Chi-Un Pae, MD, PhD

Department of Psychiatry, Bucheon St. Mary's Hospital, The Catholic University of Korea College of Medicine, 327 Sosa-ro, Wonmi-gu, Bucheon 420-717, Republic of Korea

Tel: +82-32-340-7067, Fax: +82-32-340-2255, E-mail: pae@catholic.ac.kr

(a) This is an Open Access article distributed under the terms of the Creative Commons Attribution Non-Commercial License (http://creativecommons.org/licenses/bync/3.0) which permits unrestricted non-commercial use, distribution, and reproduction in any medium, provided the original work is properly cited. gous to CREB, with which regulates synaptogenesis and spontaneous neural network activity. ${ }^{4}$ Furthermore, CREM has been also found to be associated with physiological and developmental role within the hypothalamic-pituitary-gonadal axis, ${ }^{5}$ neuronal degeneration and plasticity, ${ }^{6}$ where are implicated in the treatment response of affective disorders. ${ }^{7}$ To date, increasing emphasis has recently been placed upon the investigation of possible epistatic interactions between genetic polymorphisms in candidate genes possibly influencing treatment outcomes. ${ }^{8-10}$ Epistasis could be defined as the functional interaction between genes. It encompasses various events including promoter activity control, chromatin remodeling, epigenetic control, as well as many other molecular reactions. ${ }^{11}$ Because these events can impact cell lifecycles and complex traits and are orchestrated through genetically driven complex yet flexible activities, the research for epistatic interactions between different candidate genes could represent a significant advantage in comparison to the investigation of single genes as a way to understand the biologic diversity that could influence, for instance, drug response. ${ }^{11}$

Accordingly, the aim of the present work is to investigate the existence of possible epistatic interactions influencing mood 
Table 1. Influence of rs6740584 within CREB and rs12775799 within CREM on clinical and socio-demographic outcomes in the present study

\begin{tabular}{|c|c|c|c|c|c|c|c|}
\hline \multirow[t]{2}{*}{$\begin{array}{l}\text { Clinical and demographic } \\
\text { characteristics of the sample }\end{array}$} & \multirow[t]{2}{*}{$\begin{array}{c}\text { Bipolar } \\
\text { disorder patients } \\
\quad(\mathrm{N}=132)\end{array}$} & \multicolumn{2}{|c|}{$\begin{array}{l}\text { Influence of } \\
\text { rs6740584 }\end{array}$} & \multicolumn{2}{|c|}{$\begin{array}{l}\text { Influence of } \\
\text { rs12775799 }\end{array}$} & \multicolumn{2}{|c|}{$\begin{array}{l}\text { Influence of the epistatic } \\
\text { interaction between } \\
\text { rs6740584 and rs12775799 }\end{array}$} \\
\hline & & $\beta$ & $\mathrm{p}$-value & B & $\mathrm{p}$-value & $\beta$ & $\mathrm{p}$-value \\
\hline \multicolumn{8}{|l|}{ Gender } \\
\hline Males & $87(66 \%)$ & 0.42 & 0.07 & 0.46 & 0.03 & -0.54 & 0.05 \\
\hline Age & $36.35 \pm 11.60$ & 0.14 & 0.55 & 0.06 & 0.76 & -0.24 & 0.38 \\
\hline YMRS baseline & $33.27 \pm 9.09$ & -0.15 & 0.51 & -0.03 & 0.86 & 0.02 & 0.94 \\
\hline YMRS discharge & $19.80 \pm 5.25$ & -0.08 & 0.72 & 0.04 & 0.83 & -0.03 & 0.90 \\
\hline YMRS \% improvement & $37.14 \pm 19.26$ & 0.08 & 0.72 & 0.13 & 0.49 & -0.13 & 0.63 \\
\hline \multicolumn{8}{|l|}{ Response } \\
\hline Yes & $33(25 \%)$ & 0.24 & 0.30 & 0.27 & 0.17 & -0.27 & 0.33 \\
\hline \multicolumn{8}{|l|}{ Remission } \\
\hline Yes & $10(8 \%)$ & 0.08 & 0.72 & 0.07 & 0.73 & -0.07 & 0.78 \\
\hline \multicolumn{8}{|l|}{ Clinical subtypes } \\
\hline $\mathrm{BD}$ without $\mathrm{PF}$ & $73(55 \%)$ & -0.40 & 0.08 & -0.34 & 0.07 & 0.59 & 0.04 \\
\hline BD with PF & $57(43 \%)$ & & & & & & \\
\hline Age at onset & $26.58 \pm 10.19$ & 0.39 & 0.11 & 0.29 & 0.14 & -0.54 & 0.07 \\
\hline \multicolumn{8}{|c|}{ Familiar history of psychiatric disorders } \\
\hline Yes & $42(32 \%)$ & 0.19 & 0.52 & -0.18 & 0.46 & 0.23 & 0.50 \\
\hline \multicolumn{8}{|l|}{ Suicide attempts } \\
\hline Yes & $22(17 \%)$ & -0.19 & 0.50 & -0.10 & 0.62 & 0.26 & 0.41 \\
\hline Duration of admission (days) & $33.66 \pm 21.07$ & 0.02 & 0.93 & 0.14 & 0.50 & -0.11 & 0.71 \\
\hline \multicolumn{8}{|l|}{ Drug } \\
\hline Lithium & $41(31 \%)$ & 0.17 & 0.54 & 0.18 & 0.41 & -0.26 & 0.43 \\
\hline Valproate & $56(42 \%)$ & & & & & & \\
\hline Other & $5(4 \%)$ & & & & & & \\
\hline \multicolumn{8}{|l|}{ Concomitant anxiolytics } \\
\hline Alprazolam & $5(4 \%)$ & 0.35 & 0.19 & 0.25 & 0.26 & -0.41 & 0.21 \\
\hline Lorazepam & $82(62 \%)$ & & & & & & \\
\hline Clonazepam & $3(3 \%)$ & & & & & & \\
\hline Buspiron & 7 (5\%) & & & & & & \\
\hline None & $35(26 \%)$ & & & & & & \\
\hline
\end{tabular}

CREB: cyclic adenosine monophosphate Response Element Binding, CREM: cAMP response element-modulator, YMRS: Young Mania Rating Scale, BD: bipolar disorder, PF: psychotic features

stabilizers and antidepressants response between genetic variants within CREB and CREM that we individually investigated in a previous paper ${ }^{12}$ in $\mathrm{BD}$ and MDD patients treated with different mood stabilizers and antidepressants.

\section{METHODS}

The subjects was described in detail in a previous study. ${ }^{12}$ Briefly, 132 and 145 in-patients suffering from BD and MDD according to DSM-IV criteria ${ }^{13}$ were consecutively collected at the Department of Psychiatry of the Catholic University of Korea College of Medicine, Seoul, Korea. There was no particular restriction with regard to the specific mood stabilizers em- ployed. However, patients were excluded if they had current severe or unstable medical and neurological conditions, current treatment with a long-acting antipsychotic, concomitant alcohol and substance abuse disorders and if they were not of Korean ethnicity. All patients admitted to the hospital were assessed for the severity of illness at baseline and at discharge by means of the Young Mania Rating Scale (YMRS) and Hamilton Depression Rating Scale (HAMD). ${ }^{14,15}$ In accordance with previous studies, response was a priori defined as a $\geq 50 \%$ symptoms' reduction from baseline to discharge. ${ }^{16}$ Remission was defined as a YMRS score $\leq 12$ and HAMD score $\leq 7$ at discharge, respectively. ${ }^{17}$ As an example, Table 1 represent the case of bipolar patients. The case of MDD is not presented due 
to space limitation (data available on request). The study protocol was approved by the institutional review board (approval number HC10TISI0031).

The main outcome measure of the present study was the existence of possible epistatic interactions between rs6740584 within CREB [position=208429351(8922), alleles $=\mathrm{C} / \mathrm{T}$, location=intron)] and rs12775799 within CREM \{[position= 35488264 (61501), alleles=C/T, location=intron] $\}$ and YMRS and HAMD improvement scores. Secondary outcome measures included the investigation of epistatic interactions between the same two SNPs and other clinical and socio-demographical variables included in the present study. The two SNPs mentioned above were selected in accordance with the following criteria: 1) among SNPs available in our dataset, ${ }^{12} 2$ ) we chose 1 SNP for each gene, 3 ) that tagged the largest number of SNPs within the same gene, so as to cover a portion of the gene as high as possible (data from http://hapmap.ncbi. nlm.nih.gov/). For CREB, only one SNP was available. ${ }^{12}$ For CREM, we choose rs12775799 because it was the SNP that allowed us to cover the larger part of the gene (approximately $14 \%)$.

Statistical analyses were performed using 'Statistica' package. ${ }^{18} \mathrm{~A}$ multiple regression model was employed to investigate the existence of possible epistatic interactions between the two genotypes and clinical/socio-demographical variables included in the present study. Clinical improvement was calculated based on changes from baseline to the end of treatment.

All p-values were 2-tailed and statistical significance was conservatively set at the 0.003 level (approximately corresponding to the 14 variables outlined in Table 1 ). With these parameters we had a sufficient power $(0.80)$ to detect a medium-large ( $\mathrm{d}=0.3$ ) effect size for patients carrying the TT rs 6740584 genotype as compared with those carrying the CT genotype. ${ }^{19}$ Such effect size corresponded to the possibility of detecting final differences on YMRS as high as 2 points.

\section{RESULTS}

No significant epistatic interaction between rs6740584 within CREB and rs12775799 within CREM on YMRS improvement measured by the change in YMRS total scores from baseline (admission) to the endpoint (discharge) was observed $(\beta=-0.13, p=0.63)$. In the analysis of secondary outcome measures (total score on YMRS at baseline and discharge, response, remission, subtypes of $\mathrm{BD}$, age, gender, age at onset, family history of psychiatric disorders, duration of admission, suicide attempt, mood stabilizers, and concomitant anxiolytics), no significant epistatic interactions between rs6740584 within CREB and rs12775799 within CREM were observed as well (all p-values $>0.003$ ). Similar trends were also observed in
HAMD-related outcomes and clinical factors in patients with MDD. A multiple regression results are presented in Table 1 (BD data only and MDD data available on request).

\section{DISCUSSION}

The present work was aimed at exploring the existence of possible epistatic interactions between rs6740584 within CREB and rs12775799 within CREM on symptom improvement scores and on other clinical variables in a sample of $\mathrm{BD}$ and MDD patients. The results of our study point to the lack of significant epistatic interactions between the two SNPs and clinical improvement, suggesting a lack of association between these genetic variants and clinical improvement in BD and MDD patients. Note, however, that our finding could also be due to the moderately small sample size of our sample that could not allow us to detect subtle differences that are usually associated with single genes or gene-gene interactions in complex disorders. ${ }^{20}$

It is also worth mentioning that in our earlier paper focusing in single SNPs within CREB and CREM no significant association with YMRS scores was observed as well. ${ }^{12}$ However, there is some evidence so far to suggest that epistatic interactions can be observed even when single SNPs individually considered do not lead to positive findings. ${ }^{21}$ Such a possibility, in turn, raises significant computational and theoretical difficulties when considering all possible gene-gene interactions not to mention other forms of genetic expression controls. ${ }^{22}$ Note also that no further significant epistatic interactions on any of the secondary outcome measures have been observed. These findings are in line with earlier findings observed in our original study. ${ }^{12}$ Moreover, they extend those findings by suggesting that no epistatic interaction exists between the two SNPs. Not surprisingly, no interaction effects between CREB and CREM on phenotypes and treatment outcomes were also seen in patients with MDD in our further analysis. Hence, we may assume that these two variants do not have epistatic interaction at least in our patients with affective disorders.

Before firm conclusions are drawn, several limitations affecting the present study should be considered. ${ }^{23}$ First of all, the lack of associations observed in the present study could be simply due to the lack of statistical power. This, in turn, could obscure small effects exerted by single SNPs. This issue is particularly concerning if one considers that even among pharmacogenetic studies with a large sample size, results tend to remain conflicting. ${ }^{24} \mathrm{~A}$ further concern is related to the use of mood stabilizers with different mechanisms of action that do not allow to reach definitive conclusions with regard to the influence of the SNPs on single mood stabilizers. ${ }^{25}$ However, our decision to include patients treated with different drugs 
could have the advantage of being closer to "real world" clinical practice. In addition, the duration of hospitalization in the present study could be considered as insufficient to ascertain a lack of response and remission. However, this time frame is consistent with common clinical practice. ${ }^{26}$ Furthermore the different duration of hospitalization could raise concerns about the fact that clinical improvement could vary as a function of time rather than of genetic variants. However, we checked for such a possibility, finding no significant influence. Finally, a further limitation of this study could be related to the incomplete coverage of genes due to our tagging approach.

Epistasis between different genes still represents a particularly relevant analysis to be performed in genetic association studies till today, being helped by the knowledge of the principal ways by which the so-called reactome displays its activities. ${ }^{9,10}$ The inclusion of the analysis of the epistasis between genes along with the analysis of the impact of single genotypes and haplotypes should be considered as a standard in the next association studies. ${ }^{9,10}$ When epistasis findings are widely available and collected across research teams over the world, such data will also substantially contribute to the understanding of the role of gene-gene interaction in affective disorders and establishment of personalized-medicine for difficult-totreat patients. However, our findings preliminary suggest that no epistatic interactions exist between CREB and CREM and clinical outcomes in BD and MDD patients treated with different antidepressants. Thus, further researches may investigate whether our results hold in a larger sample of BD and MDD patients treated with different antidepressants or whether the investigation of different SNPs and/or different genegene interactions within other genes involved in the transcription pathways might lead to different outcomes.

\section{Acknowledgments}

This study was supported by a grant of the Korean Health Technology R\&D Project, Ministry of Health \& Welfare, Republic of Korea (HI12C0003).

\section{REFERENCES}

1. Silva AJ, Kogan JH, Frankland PW, Kida S. CREB and memory. Annu Rev Neurosci 1998;21:127-148.

2. Wilkie MJ, Smith D, Reid IC, Day RK, Matthews K, Wolf CR, et al. A splice site polymorphism in the G-protein beta subunit influences antidepressant efficacy in depression. Pharmacogenet Genomics 2007; 17:207-215.

3. Mamdani F, Alda M, Grof P, Young LT, Rouleau G, Turecki G. Lithium response and genetic variation in the CREB family of genes. Am J Med Genet B Neuropsychiatr Genet 2008;147B:500-504.

4. Aguado F, Diaz-Ruiz C, Parlato R, Martinez A, Carmona MA, Bleckmann $\mathrm{S}$, et al. The CREB/CREM transcription factors negatively regulate early synaptogenesis and spontaneous network activity. J Neurosci 2009;29:328-333.

5. Sassone-Corsi P. Coupling gene expression to cAMP signalling: role of CREB and CREM. Int J Biochem Cell Biol 1998;30:27-38.

6. Mantamadiotis T, Lemberger T, Bleckmann SC, Kern H, Kretz O, Mar- tin Villalba A, et al. Disruption of CREB function in brain leads to neurodegeneration. Nat Genet 2002;31:47-54.

7. Lee KS, Park YM, Lee SH. Serotonergic dysfunction in patients with bipolar disorder assessed by the loudness dependence of the auditory evoked potential. Psychiatry Investig 2012;9:298-306.

8. Chiesa A, Lia L, Han C, Lee SJ, Pae CU, Serretti A. Investigation of epistasis between DAOA and 5HTR1A variants on clinical outcomes in patients with schizophrenia. Genet Test Mol Biomarkers 2013;17:504507.

9. Pae CU, Drago A, Patkar AA, Jun TY, Serretti A. Epistasis between a set of variations located in the TAAR6 and HSP-70 genes toward schizophrenia and response to antipsychotic treatment. Eur Neuropsychopharmacol 2009;19:806-811.

10. Pae CU, Drago A, Forlani M, Patkar AA, Serretti A. Investigation of an epistastic effect between a set of TAAR6 and HSP-70 genes variations and major mood disorders. Am J Med Genet B Neuropsychiatr Genet 2010;153B:680-683.

11. Phillips PC. Epistasis--the essential role of gene interactions in the structure and evolution of genetic systems. Nat Rev Genet 2008;9:855-867.

12. Crisafulli C, Shim DS, Andrisano C, Pae CU, Chiesa A, Han C, et al. Case-control association study of 14 variants of CREB1, CREBBP and CREM on diagnosis and treatment outcome in major depressive disorder and bipolar disorder. Psychiatry Res 2012;198:39-46.

13. Sheehan DV, Lecrubier Y, Sheehan KH, Amorim P, Janavs J, Weiller E, et al. The Mini-International Neuropsychiatric Interview (M.I.N.I.): the development and validation of a structured diagnostic psychiatric interview for DSM-IV and ICD-10. J Clin Psychiatry 1998;59(Suppl 20): 22-33.

14. Young RC, Biggs JT, Ziegler VE, Meyer DA. A rating scale for mania: reliability, validity and sensitivity. Br J Psychiatry 1978;133:429-435.

15. Hamilton M. A rating scale for depression. J Neurol Neurosurg Psychiatry 1960;23:56-62.

16. Hirschfeld RM, Keck PE Jr, Kramer M, Karcher K, Canuso C, Eerdekens $\mathrm{M}$, et al. Rapid antimanic effect of risperidone monotherapy: a 3-week multicenter, double-blind, placebo-controlled trial. Am J Psychiatry 2004;161:1057-1065.

17. Riedel M, Moller HJ, Obermeier M, Schennach-Wolff R, Bauer M, Adli $\mathrm{M}$, et al. Response and remission criteria in major depression--a validation of current practice. J Psychiatr Res 2010;44:1063-1068.

18. StatSoft I. STATISTICA per Windows, 1995.

19. Cohen J. Statistical Power Analysis for the Behavioral Sciences. Hillsdale, New Jersey: Lawrence Erlbaum Associates; 1988.

20. Risch N, Herrell R, Lehner T, Liang KY, Eaves L, Hoh J, et al. Interaction between the serotonin transporter gene (5-HTTLPR), stressful life events, and risk of depression: a meta-analysis. JAMA 2009;301:24622471.

21. Arias B, Aguilera M, Moya J, Saiz PA, Villa H, Ibáñez MI, et al. The role of genetic variability in the SLC6A4, BDNF and GABRA6 genes in anxiety-related traits. Acta Psychiatr Scand 2012;125:194-202.

22. Serretti A, Chiesa A. The challenge of uncovering the genetics of anxiety: an editorial comment to Arias B, Aguilera M, Moya J et al. 'The role of genetic variability in the SLC6A4, BDNF and GABRA6 genes in anxiety-related traits' (2). Acta Psychiatr Scand 2012;125:185-186.

23. Pae CU. Strategies to find candidate genes for bipolar disorder. Psychiatry Investig 2009;6:316.

24. Shi J, Badner JA, Gershon ES, Liu C. Allelic association of G72/G30 with schizophrenia and bipolar disorder: a comprehensive meta-analysis. Schizophr Res 2008;98:89-97.

25. Kim B, Lim JH, Kim SY, Joo YH. Comparative Study of Personality Traits in Patients with Bipolar I and II Disorder from the Five-Factor Model Perspective. Psychiatry Investig 2012;9:347-353.

26. Zimmerman M, Mattia JI, Posternak MA. Are subjects in pharmacological treatment trials of depression representative of patients in routine clinical practice? Am J Psychiatry 2002;159:469-473. 